\title{
Effect of Alkali Treatment on the Dyeing and Comfort properties of Modified Polyester fabric
}

\author{
R.Rathinamoorthy* \\ \{r.rathinamoorthy@gmail.com\} \\ Department of Fashion Technology, PSG College of Technology, Coimbatore, India
}

\begin{abstract}
In this research, an attempt has been made to study the effect of alkali treatment on the dyeing ability of the commercially available modified polyester fabric. The modified polyester was treated with different concentrations of alkali namely 5,10 , and $15 \%$. The treated polyester fabrics were evaluated for the colour strength $(\mathrm{K} / \mathrm{S})$ value and comfort properties. The result indicates that the alkali treatment improved the colour strength value considerably for reactive dyes. In the case of natural dye, the colour strength value significantly reduces after subsequent alkali treatment. It is also evident that the alkali treatment considerably reduced the fabric tensile strength. But it has less influence on the tearing strength of the fabric and wickability. However, the alkali treatment increased the water absorbency, water vapour permeability, and air permeability values than the normal modified fabric significantly. Hence, this study suggests that alkali treatment of the modified polyester considerably improves the comfort properties and dye uptake of the alkali-treated fabric, except for the strength factor. Still, further studies need to be done for the optimization of the alkali concentration to restrict the strength reduction in fabric.
\end{abstract}

Keywords: Modified polyester fabric, Alkali treatment, Colour strength value, Physical properties.

\section{Introduction}

Textile materials are one of the essential requirements of human life. Mainly the textiles materials used to protect a person's body from the environment. Later the clothing materials started to several other purposes like comfort, look, and style. Out of this, clothing comfort is one of the very important parameters that consumer looks into the textile produces. Comfort refers to the condition of the mind which expresses satisfaction concerning the environment. The comfort of the human body is influenced by several factors like physical, physiological, and psychological aspects [1]. Due to its cheaper price and versatile properties, polyester fiber becomes the one of most commercially accepted fibers in the market. The long-life, easy-care properties, and versatile application potential of Polyester fabrics have attracted a wide range of commercial and industrial customers [2]. In the meantime, polyester and other synthetic textile fibers were often criticized for their poor handle, moisture, and thermal properties [3]. Several researchers reported that the surface modification of synthetic textiles is one of the potential ways to modify or to improve those properties successfully [3].

Exposure of polyester textiles to alkaline or acids or certain solvents has a significant impact on the surface chemical structure. These changes increase the amorphous region of the polyester fiber and help in improving the dyeability and moisture-related characteristics [4]. 
There are several researchers studied the effect of alkali on polyester [5-8]. The effect of alkaline hydrolysis treatment on the degradation of polyester geotextile has been investigated by Rahman [9]. Sato [10], Kim et al [11], and Zeronian [12] et al carried out work on alkaline hydrolysis and enzymatic treatments of different types of polyester fibers. Gorchakova et al [13] have studied the effect of structural modification of polyester fiber by alkaline hydrolysis. Nowadays there are different physically and chemically modified polyester fabrics were available in the commercial market. The main advantage of the modified polyester fabric is that the fabrics can able to provide a comfort feel like cotton and strength like synthetic fibers. The physical and chemical treatment imparted on the polyester fabric is the main reason for their improved properties [14]. In this work, the effect of alkali treatment on the dyeing and comfort properties of the commercially available modified polyester fabric was studied. In this study, the dyeing of modified polyester fabric with reactive and natural dye is done before and after alkali treatment. The colour values were analyzed using a spectrophotometer. Further, the influence of alkali treatment on the physical properties, comfort characteristics, and also on colour strength $(\mathrm{K} / \mathrm{S})$ values were evaluated.

\section{Materials and methods}

Materials

The commercially available modified polyester fabric is purchased from the retail stores of Coimbatore. The plain-woven modified polyester fabric with 96 ends per inch and 124 picks per inch. The reactive dye is obtained from Hi-pure chemicals, Mumbai. Sodium hydroxide required for alkali treatment is also obtained from Sigma-Aldrich, USA. All the chemicals used were of analytical grade without any purification. Commercially available Natural dye was purchased from the retail outlets of Coimbatore. The purchased dye was used as such with the same analytical grade.

\section{Alkali treatment}

Alkali-treatment followed by immersing the samples in a sodium hydroxide solution whose concentration is $5 \%(\mathrm{w} / \mathrm{w})$ at $50: 1$ liquor ratio. The initial temperature was $40{ }^{\circ} \mathrm{C}$ and the temperature was raised to boil for $60 \mathrm{~min}$. The procedure is repeated for the different concentrations like $10 \%$ and $15 \%$ concentrations of sodium hydroxide

Dyeing of modified polyester

Deep shade dyeing is done for modified fabric treated after different $\mathrm{NaOH}$ concentrations. For the treatment, a natural dye and a reactive dye were used with the following recipe. Dye - $2 \%$ On weight of material (owm); $\mathrm{NaCl}$ (first-45 min) - 80 grams per liter (gpl); $\mathrm{NaOH}$ (second-45 min) - 23 gpl; MLR - 1:20; Temperature - 80 C; Time - 90 min

\section{Dyeing of modified polyester using natural dye}

Similarly, the deep shade dyeing is done for various concentration treated fabric using natural dye. In natural dye also the dyeing is also carried out for 90 min without using any other chemicals.

Analyses of colour value using spectrophotometer 


\section{Basic Principles}

The $K / S$ values of the dyed fabric were determined by measuring their surface reflectance using a computer-aided Macbeth 2020 plus reflectance spectrophotometer followed by calculating the $K / S$ values using Kubelka Munk equation with the help of relevant software [15].

\section{Influence of alkali treatment on the physical property}

The effect of the alkali treatment on the following physical properties was studied as per the standards. Tensile strength (ASTM D5034), elongation (ASTM D5034), Tearing strength (ASTM D1424), Water absorption (BS 4554) Water vapor permeability (BS-1970), Air Permeability (ASTM D 737-96), Wicking (AATCC 79- stripe test). The values provided in the charts were the average of the triplicate of the test results.

\section{Results and discussions}

\section{Analyses of colour value}

The colour strength values of the modified polyester fabric before and after treatment were analysed and the results were given in figure 1 . The result indicates that the increment in the percentage of alkali for the treatment successively increases the dye uptake in the fabric [16]. The basic mechanism was explained by Karmakar. The number of hydroxyl groups increases due to alkali hydrolysis, but there is some damage to the fiber structure. The fiber cross-section becomes more complex and the damage cause increased surface area [17], which makes the improved dye uptake in the alkaline finished fabric.

In figure 1 , it can be seen that the $15 \%$ alkali-treated fabric shows the highest amount of $\mathrm{K} / \mathrm{S}$ value around 1.013 than the other fabrics. But in the case of the untreated fabric, the colour strength value is very less around 0.321 . This may be explained that the fiber content in the modified polyester fabric weft yarn was textured polyester and the warp was made of usual polyester. Hence, the reactive dyeing process dyed only the textured content in weft yarn in the case of untreated modified fabric. In the warp direction, the alkali treatment might have resulted fiber degradation of polyester fiber at the structural level. 


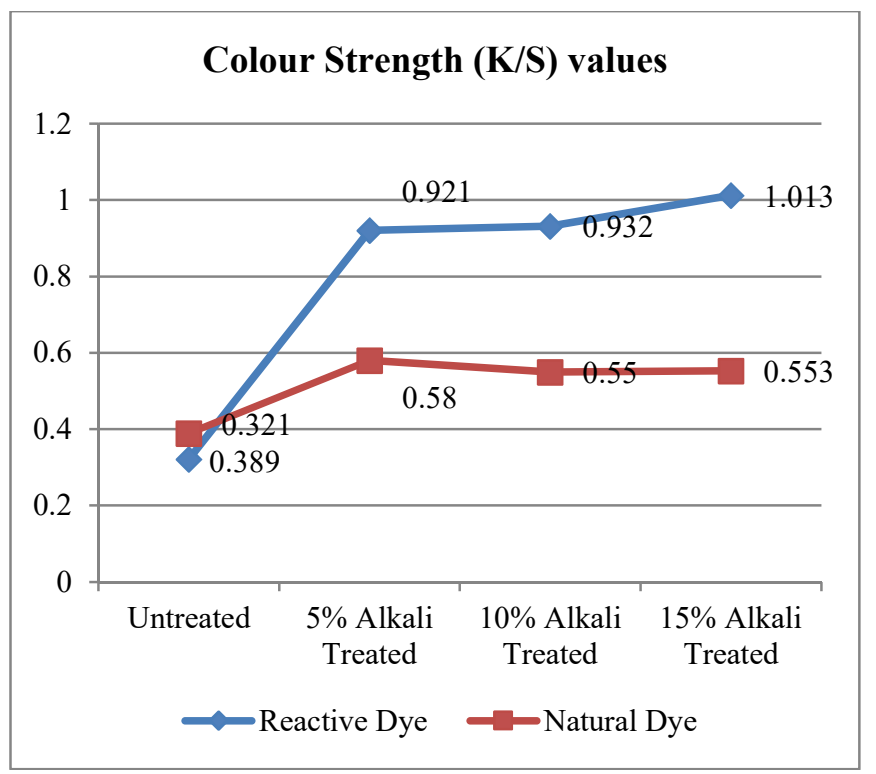

Figure 1 Colour strength $(\mathrm{K} / \mathrm{S})$ value of the alkali treated fabric

The treatment of $\mathrm{NaOH}$ hydrolysis on the polyester surface creates cleavages in the ester links of the fiber [18]. Further, due to this activity the number of polar groups in the surface of the fiber increases after alkali treatment [19]. This process generated more amorphous regions and attractive sites in the polyester fiber and hence improves the dye uptake percentage. In the case of the natural dyeing process, the colour strength value of the alkali finished fabric increased. However, the amount of dye uptake is considerably less than the reactive dye. It can be noticed from the result, the 5\% alkali treatment improved the colour strength value from 0.382 to 0.58 . But further increment the alkali percentage reduces the dye uptake percentage. In the 5\% alkali treatment, the colour strength values were higher than the untreated. For further increment in alkali concentration on fabric, the colour strength value almost remained the same or less than the $5 \%$ alkali-treated fabric.

\section{Influence of Alkali treatment on the physical properties}

\section{Tensile Strength}

The effect of alkali treatment on the tensile strength of the modified polyester fabric was evaluated. The results indicate that the $15 \%$ concentration of $\mathrm{NaOH}$ treated fabric has affected majorly. The strength value is reduced by up $14 \%$ in the warp way and $23 \%$ in the weft way. The reduction in strength value is also reflected in the elongation of the fabric. There is a $38 \%$ of increment in the elongation length in the warp direction and the weft, it is observed as a reduction in elongation. To the fiber content, the weft yarns in the fabric are textured polyester fiber. Hence, the alkali treatment has affected the properties of the textured fiber. This was explained by the drastic strength reduction in the weft yarn than the warp yarn. It was reported that the treatment of polyester fabric with $\mathrm{NaOH}$ reduced the fabric weight and breaking strength. Further, the treatment was also noted to increase the handle value of the fabric [20]. Similar results were reported by other researchers also [21 -23]. 


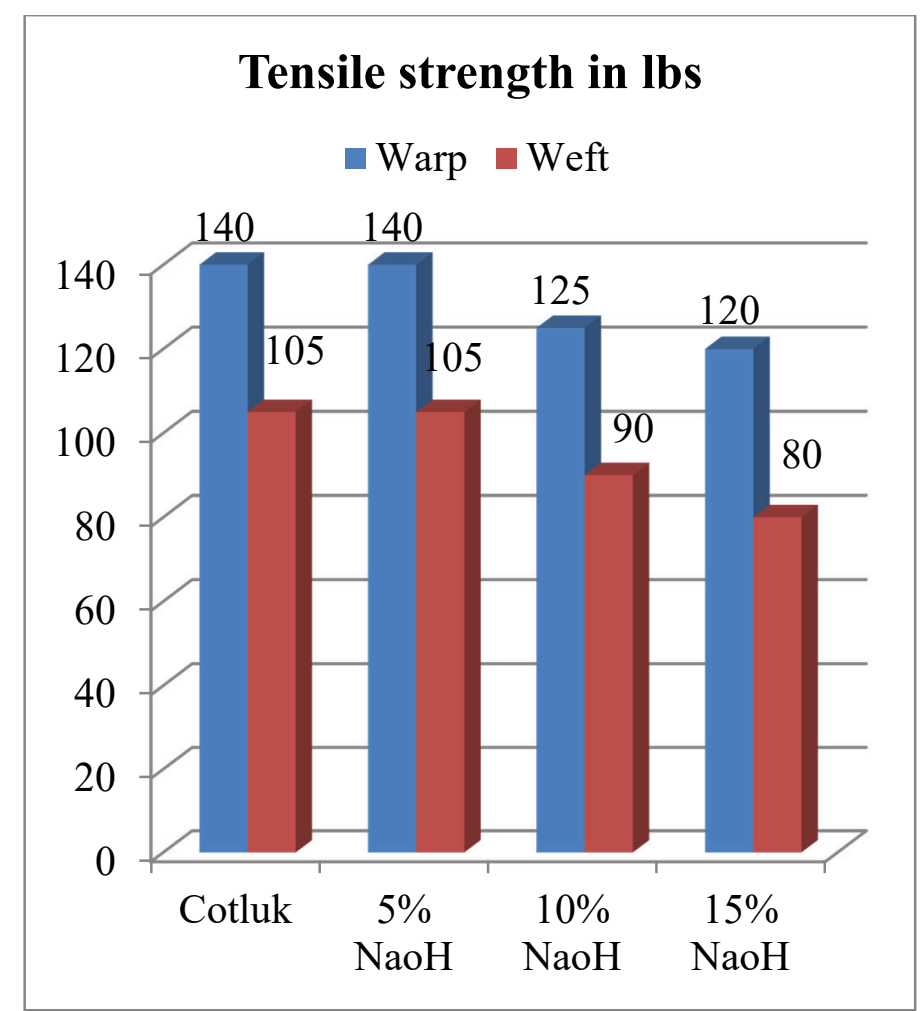

Figure 2 Tensile strength of alkali treated fabric in Ibs

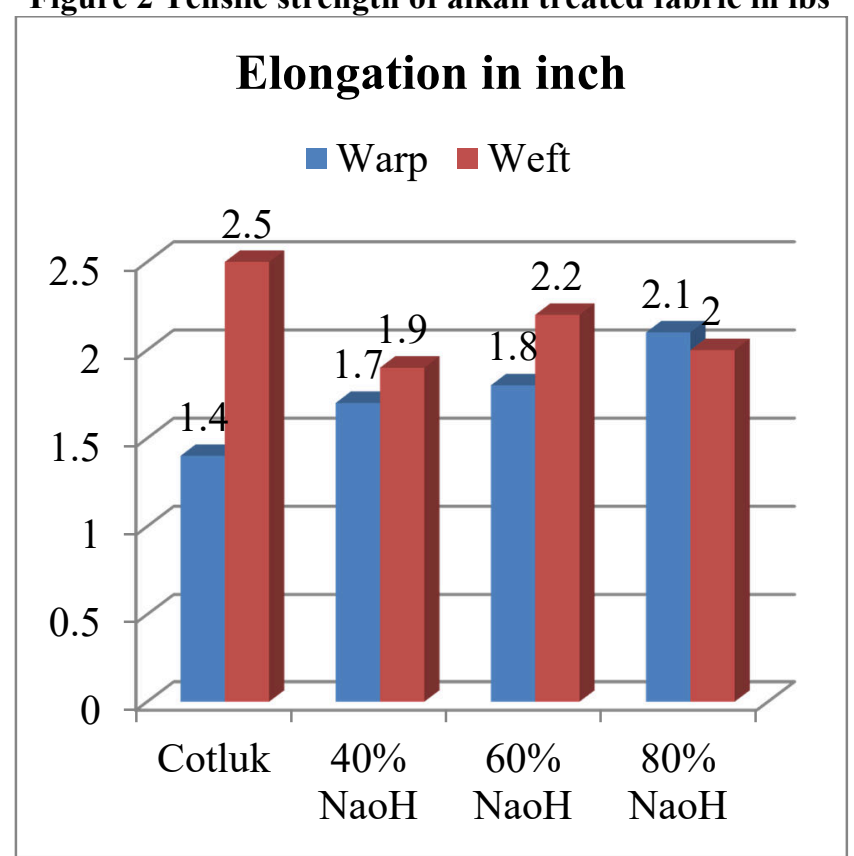

Figure 3 Elongation of alkali-treated fabric in inch 
The result shows that the alkali treatment has reduced the tearing strength value of the modified polyester fabric. However, it can be seen that, at the maximum concentration of $15 \%$ alkali, the warp way strength reduction is only $3.34 \%$. In the case of the weft way, the reduction is $4.21 \%$. This higher degradation in the weft yarn maybe because of the presence of textured fiber in the weft direction. In general, the effect of alkali treatment on the tearing strength of the fabric is considerably less than the tensile strength.

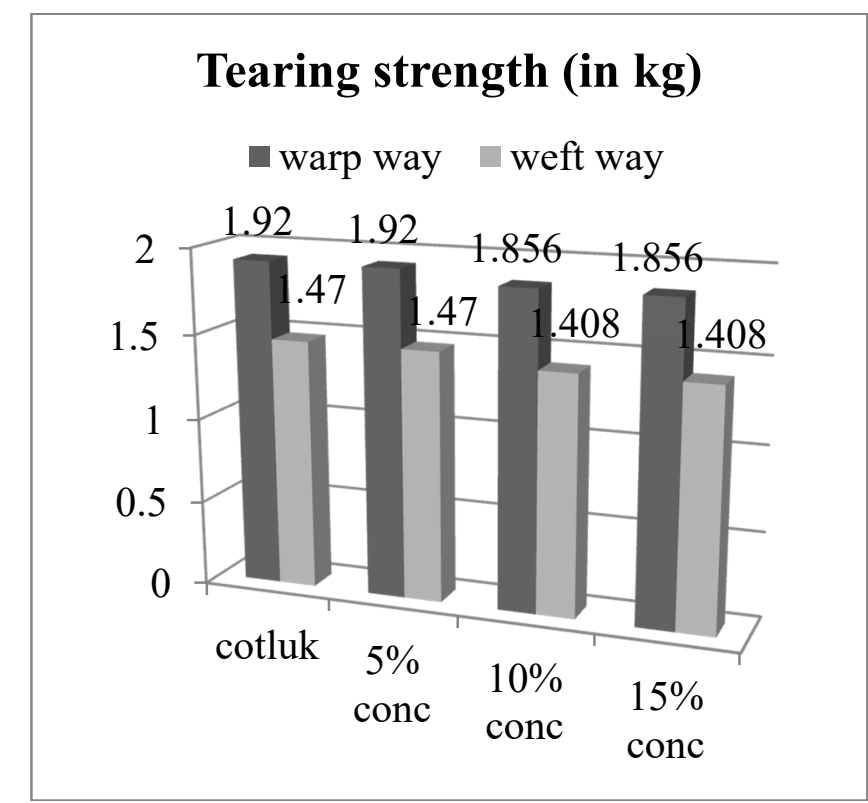

Figure 4 Tearing strength of Alkali treated fabric

Influence of Alkali treatment on the Comfort properties

Water Absorbency

The time taken by the fabric to absorb the droplet of water was measured in this experiment. The water absorbency of the fabric is increased due to the alkali treatment process. The water absorbency is raised with the increment in the concentration of $\mathrm{NaOH}$ used for the treatment. For a higher concentration of $\mathrm{NaOH}$ treatment, the water absorption time was very less. The treatment hydrolysis the hard segments of the polyester on the surface and creates new reactive sites $(-\mathrm{OH})[24]$. Further, the textured fibers in the weft yarns, the alkali treatment causes more impact to the fibers positively by improving the water-absorbing time [25-26]. 


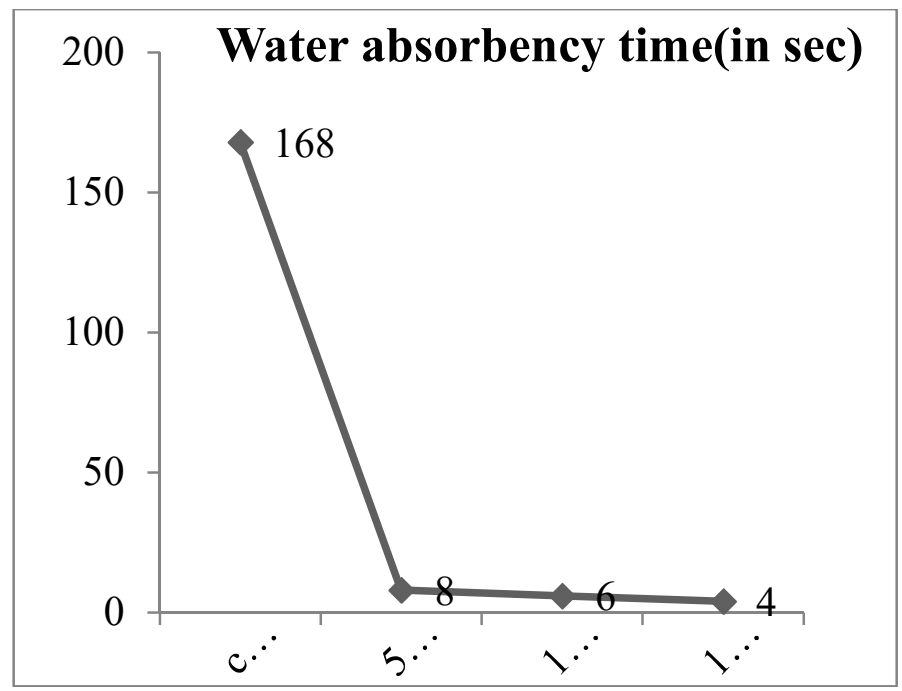

Figure 5 Water absorbency of alkali-treated fabrics

\section{Wicking}

The wicking ability of the selected fabric is comparatively high and good than the alkalitreated fabric. The result says that the fabric has a wicking height of $5.1 \mathrm{~cm}$ in warp and $4 \mathrm{~cm}$ in the weft direction at untreated state. After the treatment, based on the alkali concentration the wicking height was reduced to $3 \mathrm{~cm}$. This phenomenon can be explained because of the smooth surface and greater surface tension of the polyester fabric. In the warp direction, the fabric consists of $100 \%$ polyester yarn

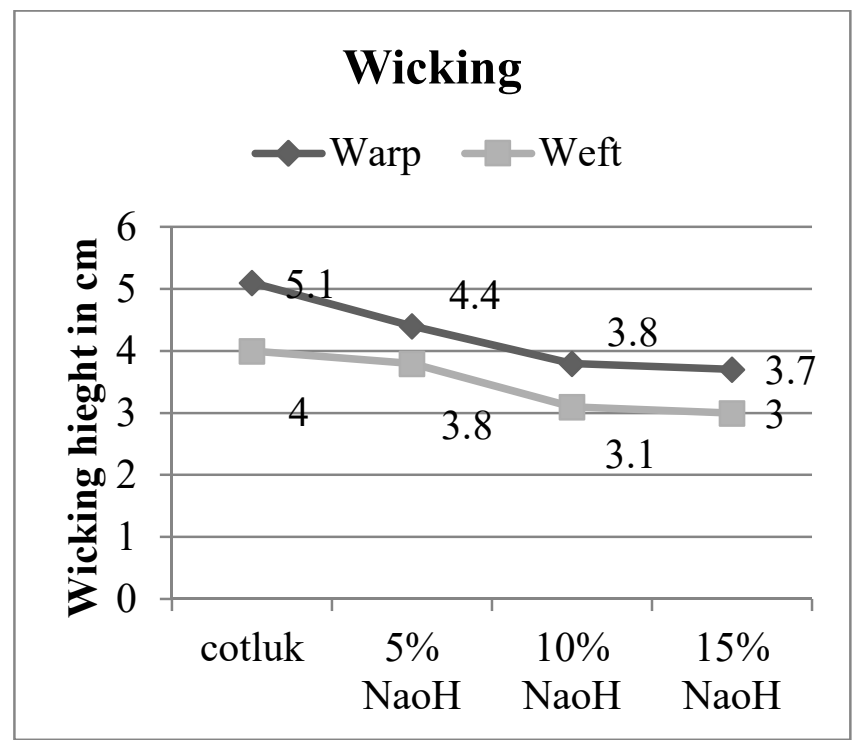

Figure 6 Wicking ability of alkali treated fabrics 
Hence, during the alkali treatment, the surface itching process affects the smooth surface and hence reduces wickability. Once the moisture is applied on the surface the development of capillary force due to the inter-fiber space drives the wicking [27]. Higher wicking results are evident here because of the non-absorbent nature of the polyester fiber. Other than this the fabric geometrical properties also play a vital role [28]. In the case of weft direction, the wicking height was less than the warp even for the fabric. This is because the weft yarn is made of textured polyester fiber blend. Hence, the cotton fiber content improves the absorbency and thus reduces the wicking. The finding of sharabaty et al. supports the fact that the hydrophilic nature of the fibers and the swelling of fibers influences the liquid flow directly [29]. During the alkali treatment, further, the surface of the existing polyester fiber also degraded and loses its surface smoothness. Hence at all the concentrations, the weft yarn wicking is less than the warp direction. These results were strongly supported by the findings of Mahish et al [30]. They had also mentioned that the alkali treatment cannot always increase the wicking or comfort behavior because unless there is a balance between wicking action and drying rate the clothing comfort can not be achieved in practical aspects [31].

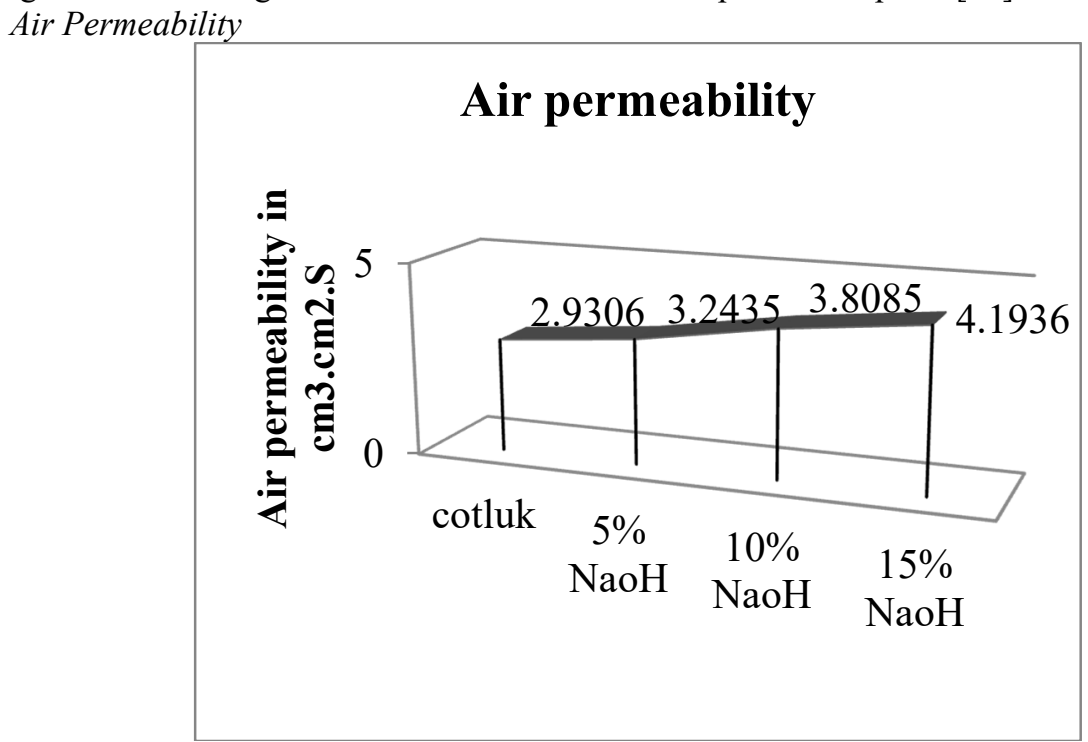

Figure 7 Wicking ability of alkali-treated fabrics

The result indicates that the increment in the alkali concentration percentage gradually increases the air permeability of the treated fabric than the untreated fabric. This may be due to the removal of part of the surface from the fibers and associated weight loss of the polyester yarn. This removal and weight loss aid more air to penetrate the structure than the untreated. Daves et al reported that the decrease in the diameter of filaments or fibers in the process of alkaline hydrolysis increases the inter-fiber and inter yarn spaces of the polyester fabric [5]. It results in a gradual increase in the porosity and consequently results in the more air permeability. These results were also in line with the findings of sudipta et al [30].

\section{Water Vapour Permeability}

Figure 8 represents the water vapour permeability values of the untreated and alkalitreated fabrics. The results indicate that the water vapour permeability of the finished fabric initially decreases for $5 \% \mathrm{NaOH}$ treatment and after that, it shows an increasing tendency. The 
initial reduction in the water vapour penetration is perhaps due to the alkali and textured fiber interaction. This process increases the yarn diameter and thus inhibits the water vapour permeability of the fabric initially. While increasing the alkali concentration further to 10 and $15 \%$, the polyester being hydrolyzed as mentioned before. Thus, the 10 and $15 \%$ treatment hydrolyses the structure and creates inter and intra fiber space. This phenomenon helps to increase the water vapour permeability after a certain stage. These results were also supported by Needles et al [32].

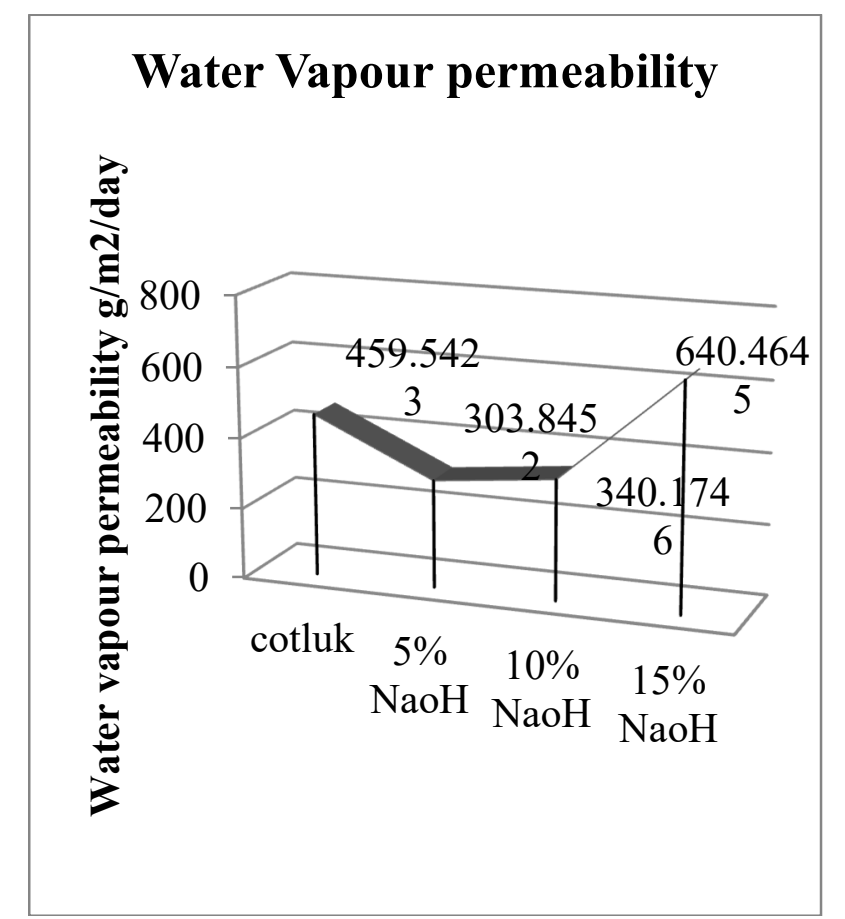

Figure 8 Water vapour permeability of alkali treated fabrics

\section{Conclusion}

This research focuses on the analysis of comfort properties of the modified polyester fabric by alkali treatment. The fabrics were treated with $5 \%, 10 \%$ and $15 \%$ concentrated sodium hydroxide and the results were evaluated. The colour strength values of the dyed fabric show that the alkali-treated fabric has higher dye uptake than the untreated. The result also shows that there is a considerable amount reduction in the tensile strength of the fabric. However, there is an increment in the few essential properties. The result highlights, there is an increase in water absorbency, water vapour permeability, and air permeability. The wicking nature of the fabric alone reduced highly after the alkaline hydrolysis process. Thus, this study enlightens the new research scope in the modified polyester sector, for improved comfort and dyeing ability. 


\section{References}

[1] Usha Rashmi Amrit, (2008) Bedding Textiles and Their Influence on Thermal Comfort and Sleep. AUTEX Research Journal, 8(4) 251-254.

[2] Olson, LM.; Wentz, M. (1984) Moisture Related properties of Hydrolysed Polyester fabrics, Text Chem Col, 16:48.

[3] Latta, BM: (1977) Clothing Comfort: Interaction of Thermal Ventilation, Construction, and Assessment Factors. Ed by NRS Hollies and RF Goldman. Ann Arbor Science, Chapter 4, Mich.

[4] Hsieh, Y. L.; Miller, A. and Thompson, J. (1996) Wetting, Pore Structure, and Liquid Retention of Hydrolyzed Polyester Fabrics. Textile Res. J., 66(1) pp. 1.

[5] Dave, J; Rajkumar and Srivastava, C.H. (1987) Studies on modification of polyester fabric by alkaline hydrolysis, J App poly sc, 33, 455-477.

[6] Teli, M.D.; Purkayastha, A. (1991) Effect of substrate geometry on alkaline Hydrolysis, American Dyestuff reporter, 80(4), 78-80.

[7] Houser, K.D. (1983) Caustic reduction of Polyester fiber, Text Chem Col, 15(4), 37-39.

[8] Teli, M.D.; Purkayastha, A. (1993) Effect of substrate geometry on alkaline Hydrolysis-Part 2, American Dyestuff reporter, 82(2), 31-40.

[9] Rahman, M. (1997) The Alkaline degradation of Polyester Geotextile, University of Leeds UK.

[10] Sato, M. (1983) Deterioration of filaments and polyethylene terephthalate with enzyme of cladosporium cladospriodes FERM J-8, Sen-I Gakkaishi, 39(5), 209-219.

[11] Kim, E.S.; Lee, C.H.; Kim, S.H. (2009) Effect of pre treatment reagents on the hydrolysis and physical properties of Pet Fabric, J App poly Sci, 112(5), 3071-3078.

[12] Zeronian, S.H.; Inglesby, M.K.; Ning pan. Lin,D.; Sun, G.; Soni, G.; Alger, K.W.; Gibbon, J.D. (1999) The fine structure of bi-component fibers, J App Poly sci, 71(7), 1163-1173.

[13] Gorchakava, M.;LZamailov, B.A.; Gartsueva, V.A. (1999) Effect of structural modification of polyester fiber on the strength of non woven material. Fiber Chem, 31(4), 315-317.

[14] Anon. (2006) As natural as it can get- Recron Perfection, Reliance News letter, 7(1),18-20.

[15] Prathiba Devi, R.; Rathinamoorthy, R.; Jeyakodi Moses, J. (2012) Effect of Jute Proportion on the Color Strength Value of Jute/Cotton Union Fabric, Int J Engg Res, 2(4), 294-299.

[16] Karmakar, S.R. (1999) Chemical Technology in the Pre-Treatment Processes of Textiles, Elsevier Publication, 12, 357.

[17] Kim, K.J. (1980) Han' guk Somyu Konghakhoechhi, 17, 151.

[18] Gorrafa A.F.M. (1980) Caustic Treatment of Polyester Filament fabrics, Text Chem Col, 12(4).

[19] Namboodri, C.G.G.; Haith, M.S. (1968) Some Aspects of Alkaline Hydrolysis of Poly

[20] (ethylene terephthalate), J Appl Poly Sci, 12, 1999.

[21] Ko S.W; Wi K.C; Kim S.Y. (1977) Sumyu Konghak Hoeji, 14,18.

[22] Achwal, W.B. (1984.) A comparative study of surface action of Caustic soda on polyester under different conditions, Man-Made Textiles India, 27,185.

[23] Timmis, J.N. (1976) Int Dyer, 156 (355) 410.

[24] Song, S.S.; Kim, S. (1983) Hanguk Somyu Konghakhoechi 20, 206.

[25] Bendak, S. M.; El-Marsafi, (1991) Effects Of Chemical Modifications On Polyester Fibres, J Islamic Acad Sci 4(4), 275-284.

[26] Anita, K.L. (2004) Bio-scouring of cotton fabrics, Ph.D. Thesis. Department of Plastics \& Rubber Technology, Budapest University of Technology \& Economics, Hungary.

[27] Ibrahim, D.F. (2014) Comparative study for Improving Printing of Cotton/Polyester Blended Fabrics. J Textile Sci Eng, 4: e119. doi:10.4172/2165-8064.1000e119

[28] Hsieh, Y.L. (1995) Liquid transport in fabric structures, Text Res J, 65(5), 299.

[29] Marchal, J.M. (2001) Modeling capillary flow in complex geometries, Text Res J, 71, 813.

[30] Sharabatya, T.; Biguenet, F.; Dupuis, D; Viallier, P. (2008) Investigation on moisture transport through polyester/cotton fabrics, Ind J Fib \& Tex Res, 33, 419-425.

[31] R. Abirami, D.S. Vijayan, Sijo Joseph John, Aldrin Albert, and Alfred Koshy Alex, Experimental Study on Concrete Properties Using Pineapple Leaf Fiber, International Journal of Advanced Research in Engineering and Technology, 11(6), 2020, pp. 913-920, DOI: 10.34218/IJARET.11.6.2020.082 
[32] Sule, A.D.; Baradhan, M.K.; Sarkar, R.K.; (2004) Development of Sportswear for Indian Conditions, Man Made Text India, 41(12), 123.

[33] Needles, H.L.; Brook, D.B., (1985) How Alkali Treatments Affect Selected Properties Of Polyester, Cotton and Polyester/Cotton Fabrics. Textile Chemist \& Colorist . 17(9) 23-26. 